\title{
Comparative Study of Glyceryl Behenate or Polyoxyethylene 40 Stearate-Based Lipid Carriers for Trans-Resveratrol Delivery: Development, Characterization and Evaluation of the In Vitro Tyrosinase Inhibition
}

\author{
Naiara Fachinetti, ${ }^{1}$ Roberta Balansin Rigon, ${ }^{1}$ Josimar O. Eloy, ${ }^{1,2}$ Mariana Rillo Sato, ${ }^{1}$ \\ Karen Cristina dos Santos, ${ }^{1}$ and Marlus Chorilli ${ }^{1,2}$
}

Received 18 September 2017; accepted 21 January 2018; published online 5 February 2018

\begin{abstract}
Trans-resveratrol (RSV) is a natural compound with several properties, such as the ability to inhibit the tyrosinase enzyme, with potential application as a skin-lightning agent and for the treatment of skin disorders associated with hyperpigmentation and melanogenesis. However, the drug faces several drawbacks which altogether limit its therapeutic application. Thus, drug loading into nanocarriers emerge as an alternative to circumvent these problems. Herein, nanostructured lipid carriers (NLCs) have been employed for RSV encapsulation, with comparison of two different lipids, glyceryl behenate (more hydrophobic), and polyoxyethylene 40 (PEG 40) stearate. PEG 40 stearate-containing NLCs presented smaller particle size and polydispersity compared with glyceryl behenate, attributed to better emulsification and nanoparticle formation, resulting in higher RSV encapsulation efficiency. Drug was loaded in both carriers as a molecular dispersion. Furthermore, the formulations had very low RSV release, which occurred due to the crystallinity degree of lipid matrix, in accordance with the DSC data. Moreover, RSV cytotoxicity against L-929 cells was not increased when loaded into nanocarriers. Interestingly, RSV-loaded formulation prepared with PEG-40 stearate resulted on greater tyrosinase inhibition than RSV solution and formulation containing glyceryl behenate, equivalent to 1.31 and 1.83 times higher, respectively, demonstrating that the incorporation of RSV into NLC allowed an enhanced tyrosinase inhibitory activity. Overall, the results obtained herein evidence potential for future in vivo evaluation of RSV-loaded NLCs.
\end{abstract}

KEY WORDS: glyceryl behenate; nanostructured lipids carriers; polyoxyethylene 40 stearate; transresveratrol; tyrosinase.

\section{INTRODUCTION}

Human skin can suffer from photoaging due to sun exposure, causing several effects such as dryness, wrinkles, elastosis, and telangiectasia, besides leading to hyperpigmentation because of melanin synthesis and accumulation increase [1, 2]. Furthermore, ultraviolet (UV) light exposure is correlated with skin cancer occurrence, which represents a major public health issue [3]. Type A UV radiation causes mitochondrial mutation and induction of matrix metalloproteinases, whereas type B UV radiation is responsible for sunburn, inflammation, and induction of oxidative stress with singlet oxygen and free radical participation [4].

\footnotetext{
${ }^{1}$ School of Pharmaceutical Sciences, Campus Araraquara, Department of Drugs and Medicines, UNESP-São Paulo State University, Rodovia Araraquara-Jau, km 1, Araraquara, São Paulo, 14800-903, Brazil.

${ }^{2}$ To whom correspondence should be addressed. (e-mail: josimar.eloy@gmail.com; chorilli@fcfar.unesp.br)
}

Regarding the mechanism of skin hyperpigmentation induced by sun exposure, the process initiates with melanin synthesis from tyrosine catalyzed by tyrosinase [5]. This coppercontained multi-functional oxidase catalyzes hydroxylation of Ltyrosine to L-DOPA and further oxidation of L-DOPA to dopaquinone. Thus, the most frequently employed approach for skin lightening involves the inhibition of tyrosinase [6, 7]. Within this context, trans-resveratrol (RSV), a widely distributed natural compound, found in grapes (Vitis vinifera L), has been demonstrated to prevent and treat skin photoaging due to its anti-inflammatory and antioxidant properties [3]. Moreover, RSV acts as melanogenesis inhibitor [7].

Although its promising use, RSV faces drawbacks, including low bioavailability due to the limited aqueous solubility and particularly instability under light and heat exposure, which hampers its use in a formulation [3, 8]. Topical administration could conveniently localize RSV on its target and previous reports indicate that traditional vehicles have been employed for RSV topical delivery [9]. Noteworthy, RSV shortcomings could be overcome through 
loading into nanostructured delivery systems, which can promote sustained drug release, protect drug from degradation triggered by light or temperature, with great potential in the cosmetic and pharmaceutical fields [10]. Within this context, nanoparticles can be prepared with different compositions with a wide sort of materials, including lipid, polymeric, and inorganic materials. Lipid nanoparticles have particular interest due to their several advantages for topical therapy, including biocompatibility and stability. First generation of solid lipid nanoparticles are composed with solid lipids at room temperature, while second generation of lipid nanoparticles, also known as nanostructured lipid carriers (NLCs), are composed by a mixture of liquid and solid lipids and are able to encapsulate drug with higher efficiency. Moreover, stability of loaded drug in NLCs is usually better [11]. Additionally, NLCs have been already shown as better RSV carriers compared with solid lipid nanoparticles, resulting in higher encapsulation efficiency and deeper skin penetration [12].

RSV has been loaded into solid lipid nanoparticles for several purposes, including oral, brain, and cutaneous delivery [13-15]. Teskec and Kristl (2010) showed that solid lipid nanoparticles caused higher uptake of RSV in keratinocytes, which could suggest their potential for topical application [16]. Furthermore, RSV and curcumin have been co-loaded in lipid-core nanoparticles for skin delivery [17]. Previously, our group synthesized RSV-loaded solid lipid nanoparticles for skin delivery; however, NLCs are likely more promising for RSV delivery due to improved stability of these nanocarriers [18]. Recently, NLCs based on cetyl palmitate and miglyol812 were loaded with RSV [19]. Furthermore, similar formulations were evaluated regarding their role on RSV delivery to modulate human dendritic cell NLCs [20]. However, to our knowledge, NCLs based on glyceryl behenate, polyoxyethylene 40 stearate, capric/caprylic acid triglycerides, and castor oil have not been reported as RSV delivery systems. The physicochemical properties of materials could influence NLC characteristics. PEG-40 stearate is a synthetic polyethyleneglycol lipid based on stearic acid, with 18 carbons in its chain, and glyceryl behenate is a lipid formed by glycerides of 22 carbon fatty acid and behenic acid. Previous reports indicated that stearic acid was able to emulsify with both poloxamer 188 and 407 in presence of PEG 4000 for lopinavir-loaded solid lipid nanoparticle formation, whereas glyceryl behenate, more hydrophobic, only emulsified with poloxamer 407 , used herein, which has a larger proportion of more hydrophobic polyoxypropylene portion than polyoxyethylene [21-23]. Noteworthy, the use of these delivery systems for tyrosinase inhibition is an innovative aspect addressed herein. Therefore, the purpose of this work is to prepare NLCs with different compositions for RSV loading, based on glyceryl behenate or polyoxyethylene 40 stearate, characterize the formulations, investigate the cytotoxicity, and evaluate the tyrosinase inhibition activity.

\section{MATERIAL AND METHODS}

\section{Material}

For formulation preparation, Milli-Q water (Merck Millipore, Germany) was employed. Polyoxyethylene stearate (40) (PEG-40 stearate), poloxamer 407 (Pluronic $\AA$ F127), methyl, and propyl parabens were supplied by Clariant (Switzerland). Glyceryl behenate (Compritol® 888 CG ATO) was purchased from Gattefossé (France), and caprylic/capric triglycerides (CRODAMOL CCTG) was obtained from Croda (UK). RSV was supplied by Suzhou Vitajoy (China). Castor oil was purchased from Dhaymers Solvay (Brazil). HPLC grade acetonitrile and methanol supplied by J.T. Baker, USA. Tyrosinase was purchased from Sigma-Aldrich. L-929 cells (ATCC®) were employed. DMEM medium, fetal bovine serum (FBS), and trypsin were obtained from SigmaAldrich.

\section{Formulation Development}

NLCs were prepared by the high shear homogenization technique [24]. First, components of the lipid phase (PEG-40 stearate or glyceryl behenate, CCTG, and castor oil) and components of the aqueous phase (poloxamer 407, methylparaben, propylparaben, and Milli-Q water) were heated separately at $70{ }^{\circ} \mathrm{C}$. RSN $(0.1 \%)$ was added to the lipid phase. The aqueous phase was kept under stirring for methylparaben and propylparaben solubilization. After complete solubilization, the aqueous phase was dipped into the oily phase that it was heated at the same temperature to form a pre-emulsion, which was dispersed using a ultraturrax homogenizer at $10,000 \mathrm{rpm}$ for $10 \mathrm{~min}$. Afterwards, the dispersion was cooled using an ice bath for lipid crystallization and NLC formation. Twelve formulations were prepared, composed either of PEG-40 stearate or glyceryl behenate. Poloxamer concentration effect was also investigated (Table I).

\section{Physicochemical Characterization of NLCs}

\section{Determination of Particle Size and Zeta Potential}

Particle size was evaluated through dynamic light scattering technique (Zetasizer Nano NS, Malvern Instruments, Malvern, UK). Dispersions of NLCs were diluted in Milli-Q water $(10 \mu \mathrm{l} / \mathrm{mL})$. Analysis were done in triplicate using scattering angle at $90^{\circ}$, temperature at $25{ }^{\circ} \mathrm{C}$, laser wavelength at $633 \mathrm{~nm}$, and refraction index at 1.311. Zeta potential was investigated using the same equipment. Measurements were done in triplicate with diluted formulations in purified water $(10 \mu \mathrm{L} / \mathrm{mL})$, with conductivity adjusted to $50 \mu \mathrm{S} / \mathrm{cm}$ with $0.1 \% \mathrm{KCl}$. Results were reported as mean \pm standard deviation.

\section{Scanning Electron Microscopy Analysis}

Determination of morphology of NLCs was done using JEOL JSM-750OF scanning electron microscope. A drop of formulation was distributed onto a carbon grid, dried, and gold coated under vacuum. Photomicrographs were obtained using 10 and $20 \mathrm{kV}$ electron beam.

\section{Diffraction Scanning Calorimetry}

Diffraction scanning calorimetry (DSC) analysis were carried out using the Jade DSC Perkin Elmer equipment. For 
Table I. Compositions of the Formulations in the Development of NLCs

\begin{tabular}{|c|c|c|c|c|c|c|c|c|}
\hline \multirow[t]{2}{*}{ Formulations } & \multicolumn{8}{|l|}{ Components (\%) } \\
\hline & PEG-40 stearate & Glyceryl behenate & CCTG & Castor oil & Poloxamer & Methylparaben & Propylparaben & Water q.s. \\
\hline 1 & 2.07 & & 2.05 & 0.88 & 1 & 0.18 & 0.02 & 100 \\
\hline 2 & 2.07 & & 2.05 & 0.88 & 2 & 0.18 & 0.02 & 100 \\
\hline 3 & 2.07 & & 2.05 & 0.88 & 2.5 & 0.18 & 0.02 & 100 \\
\hline 4 & 2.07 & & 2.05 & 0.88 & 3 & 0.18 & 0.02 & 100 \\
\hline 5 & 2.07 & & 2.05 & 0.88 & 3.5 & 0.18 & 0.02 & 100 \\
\hline 6 & 2.07 & & 2.05 & 0.88 & 4 & 0.18 & 0.02 & 100 \\
\hline 7 & & 2.07 & 2.05 & 0.88 & 1 & 0.18 & 0.02 & 100 \\
\hline 8 & & 2.07 & 2.05 & 0.88 & 2 & 0.18 & 0.02 & 100 \\
\hline 9 & & 2.07 & 2.05 & 0.88 & 2.5 & 0.18 & 0.02 & 100 \\
\hline 10 & & 2.07 & 2.05 & 0.88 & 3 & 0.18 & 0.02 & 100 \\
\hline 11 & & 2.07 & 2.05 & 0.88 & 3.5 & 0.18 & 0.02 & 100 \\
\hline 12 & & 2.07 & 2.05 & 0.88 & 4 & 0.18 & 0.02 & 100 \\
\hline
\end{tabular}

analysis, samples were lyophilized and $5 \mathrm{mg}$ of samples were used. Samples were heated from 15 to $300{ }^{\circ} \mathrm{C}$, at a rate of $10{ }^{\circ} \mathrm{C} / \mathrm{min}$. The sample scans were obtained under $\mathrm{N}_{2}$ pressure of $2 \mathrm{kgf} / \mathrm{cm}^{2}$.

\section{Thermogravimetry Analysis}

Thermogravimetry analysis (TGA) was carried out with a PerkinElmer 4000 instrument calibrated with standard weights. The lyophilized samples were weighted and then submitted to heating from 15 to $500{ }^{\circ} \mathrm{C}$ at a rate of $10{ }^{\circ} \mathrm{C} / \mathrm{min}$, under nitrogen pressure of $2 \mathrm{kgf} / \mathrm{cm}^{2}$.

\section{Encapsulation Efficiency Determination}

For evaluation of the encapsulation efficiency of formulations, a high-pressure liquid chromatography (HPLC) method was employed using the 2695 Waters Alliance equipment, with a US/Vis photodiode array detector. After scanning, the wave length was fixed at $306.6 \mathrm{~nm}$. Experiments were conducted using a C-18 column $(250 \mathrm{~mm} \times 4,6 \mathrm{~mm}$; $5 \mathrm{um}$; Phenomenex). Mobile phase was composed of a gradient of water:acetonitrile $(75: 25, v / v)$ from 0 to $3.5 \mathrm{~min}$, water:methanol:acetonitrile $(32.5: 30: 37.5, v / v)$ from 3.6 to $5.8 \mathrm{~min}$, and water:acetonitrile $(75: 25, v / v)$ from 5.9 to $10 \mathrm{~min}$ [18]. The method was validated for linearity, selectivity, precision, accuracy, limit of detection, and limit of quantification (ICH 2005). For encapsulation efficiency determination, a method previously published was followed with modifications [25]. Briefly, total formulation was solubilized in DMSO $(1: 1, v / v)$ for nanocarrier disruption and drug solubilization, followed by dilution with acetonitrile (1:20, $v / v)$. Samples were then submitted to ultrasound bath for $10 \mathrm{~min}$ and filtered $(0.45 \mu \mathrm{m})$, using PTFE membrane filters, before quantification in HPLC. Non-encapsulated precipitate drug was separated from encapsulated drug by filtration using $0.45 \mu \mathrm{m}$ PVDF membrane filters to obtain the filtrated NLC. This sample was disrupted with DMSO, diluted with acetonitrile, and quantified by HPLC. Furthermore, considering that the unloaded RSV can be present not only as a precipitate but can be soluble due to high surfactant content in the formulation, we obtained the soluble fraction using the ultrafiltration method. For that, we employed Amicon 50.000 Da molecular-weight cut-off ultrafilters, with centrifugation at $4000 \times g$ for 15 min to separate free drug fraction in the filtrate, which was then diluted in acetonitrile prior to quantification. All the samples were quantified by HPLC. Thus, RSV encapsulation efficiency was calculated as follows:

$\mathrm{EE}(\%) \frac{\mathrm{RSV}(\text { filtrated NLC) }-\mathrm{RSV}(\text { soluble })}{\text { Total RSV }} \times 100$

\section{In Vitro RSV Release Studies}

Experiments were conducted in sextuplicate using modified diffusion cells (Franz cells). Cellulose ester membranes with pore size of $12-14 \mathrm{kDa}$ molecular weight cut-off (MWCO) were placed between the donor and recipient compartments. Thereafter, $300 \mu \mathrm{L}$ of formulations were added onto the membrane in the donor compartment. Recipient solution was composed of a $2 \%$ polysorbate 80 aqueous solution and remained under stirring (300 rpm) at 32 $\pm 2{ }^{\circ} \mathrm{C}$. Samples were withdrawn, with replenishment of fresh medium, at 5, 15 and $30 \mathrm{~min}$, and after 1, 2, 4, 6, 8, 12, 16, 20, and $24 \mathrm{~h}$ in order to evaluate the release kinetics. Quantification of RSV in the recipient compartment employed the HPLC method previously described.

\section{Determination of Tyrosinase Inhibition by RSV-Loaded Formulations}

On 96-well microplates, $10 \mu \mathrm{L}$ of tyrosinase aqueous solution ( $480 \mathrm{IU} / \mathrm{mL}), 70 \mu \mathrm{L}$ of $\mathrm{pH} 6.8$ phosphate buffer, and $70 \mu \mathrm{L}$ of tyrosine aqueous solution $(0.3 \mathrm{mg} / \mathrm{mL})$ were added $[18,26]$. Thereafter, $60 \mu \mathrm{L}$ of blank NLCs (control), RSVloaded NLCs, RSV in suspension in different concentrations $(5,10,20,40$, and $80 \mu \mathrm{g} / \mathrm{mL})$, or kojic acid solution $(5.0,4.0$, $3.0,2.5,1.25,0.625$, and $0.3125 \mu \mathrm{g} / \mathrm{mL})$, used as a positive control in different concentrations, were added. Then, microplates were incubated at $30 \pm 1{ }^{\circ} \mathrm{C}$ for $120 \mathrm{~min}$. Absorbance was measured at $490 \mathrm{~nm}$ in a microplate reader 
(Thermoplate) at time zero and at the end of the reaction. The percentage of tyrosinase inhibition (TI) was calculated as follows:

$\mathrm{TI}=\frac{(A-T 0)-(B-T 0)}{A} \times 100$

where $A$ is control absorbance, $B$ is sample absorbance, and $T 0$ is absorbance time 0 .

All the analyses were done in triplicate $(n=3)$, and data are given as the average values and standard deviations.

\section{Cytotoxicity Study}

For cytotoxicity evaluation of blank NLCs and RSVloaded NLCS, the agar overlay qualitative assay was employed [27]. For that, L-929 cell line was cultivated in DMEM medium supplemented with $10 \%$ FBS at $37{ }^{\circ} \mathrm{C}$ with $5 \% \mathrm{CO}_{2}$ in a $75-\mathrm{cm}^{2}$ flask. After reaching $75-80 \%$ confluence, cells were trypsinized and cultivated in 6-well plates for $48 \mathrm{~h}$ for monolayer formation. After $48 \mathrm{~h}$, wells were washed with pH 7.4 PBS and medium composed by $1.8 \%$ agar and DMEM (1:1) with $0.01 \%$ neutral red. Following agar solidification, sterile filter paper disks were embedded with formulations and placed at the center of each well, in triplicate. After $24 \mathrm{~h}$, the wells were macroscopically examined and the formation of a clear inhibition halo was observed and measured with a caliper. Sample cytotoxicity is correlated with the halo diameter. For instance, moderate cytotoxicity results from halo inhibition in the range of 0.5 to $1.0 \mathrm{~cm}$.

\section{Statistical Analysis}

The results were presented as mean \pm standard deviation (SD). Student's $t$ test was used, and statistical significance was set at $P \quad 0.05$.

\section{RESULTS AND DISCUSSION}

Table II shows that formulations composed by PEG-40 stearate have particle size ranging from 164.6 to $329.3 \mathrm{~nm}$, whereas formulations composed by glyceryl behenate presented considerably larger particle size, varying from 380.9 to $783.1 \mathrm{~nm}$. Therefore, not only glyceryl behenate generated larger particles, but it also resulted in higher polydispersity, ranging from $0.241 \pm 0.019$ to $0.597 \pm 0.023$, probably due to its higher lipophilicity compared with PEG-40 stearate, which may result in poor emulsification with the aqueous phase. Behanic acid is a fatty acid with a longer chain length (C22 fatty acid) than either stearic acid (C18 fatty acid). Furthermore, the longer the chain length of the former would ensure a higher degree of lipophilicity [28]. Noteworthy, the polydispersity index $(\mathrm{PdI})$ is a parameter related to particle size distribution and, in this context, PdI values higher than 0.7 indicates very broad distribution, while PdI values close to 0.2 are usually associated with more homogeneous particle size distribution [29]. Formulations NLC1 and NLC8 were not characterized because they presented phase separation right after preparation.
Particles from a colloidal dispersion can adsorb ions or contain groups which are able to confer charge to particle surface, reflected by zeta potential, which is directly related to physicochemical stability. Thus, it is desirable that the nanostructured system presents high surface charge or zeta potential, usually higher than $+30 \mathrm{mV}$ or lower than $-30 \mathrm{mV}$, once forces of repulsion tend to avoid aggregation between adjacent particles [30]. As evidenced from Table II, formulations presented zeta potential between -1.21 and $-2.25 \mathrm{mV}$. However, despite near-neutral characteristic of NLCs, stabilization was achieved with poloxamer 407, which confers stability owing to steric hindrance rather than electric repulsion [31]. In this regard, Jain et al. [32] reported the role of poloxamer adsorption onto nanoparticle surface to modify the aggregation state and impart stability to formulations. This process is largely dependent on the adsorbed amount and is influenced by polymer structure, molar mass and adsorption energy [32].

NLC 4, containing PEG-40 stearate, and NLC 7, containing glyceryl behenate were selected for further studies because they showed the smallest values of particle size and PdI of each group. Particle size was increased from $164.6 \pm 36.61$ to $225.8 \pm$ 40.99 (blank NLC 4 and RSV-loaded NLC 4, respectively), but zeta potential remained unchanged and almost neutral (Table II). For comparison, particle size of RSV-loaded NLC 7 corresponded to $386.82 \pm 26.14$. Encapsulation efficiency corresponded to $61.58 \pm 0.19$ and $44.66 \pm 0.19 \%$ for formulations containing PEG-40 stearate and glyceryl behenate, respectively, indicating that PEG-40 stearate enabled higher loading of RSV in a smaller and more homogenous formulation. The value of encapsulation efficiency of NLC based on PEG-40 stearate was comparable with RSV-loaded NLCs prepared with cetyl palmitate, miglyol-812, and polysorbate 60, previously reported [19]. One hundred micrograms of RSV was loaded into $1 \mathrm{~mL}$ of NLC suspension, therefore the drug loading in the formulations 4NLC and 7-NLC, considering the encapsulation efficiency values, corresponded to 61.52 and $44.66 \mu \mathrm{g} / \mathrm{mL}$, respectively.

Electron microscopy is useful for morphology characterization of nanostructures [10]. Microphotographs depicted in Fig. 1 showed the spherical nature with smooth surface of NLCs prepared with PEG-40 stearate, which was not compromised by RSV loading. RSV-loaded NLCs prepared

Table II. Characterization of Blank NLCs and 0.1\% RSV-loaded NLCs: Particle Size, PdI, and Zeta Potential

\begin{tabular}{lcll}
\hline NLC & Particle size $(\mathrm{nm})$ & PdI & Zeta potential $(\mathrm{mV})$ \\
\hline 2 & $308.0 \pm 225.4$ & $0.412 \pm 0.065$ & $-2.01 \pm 0.265$ \\
3 & $329.3 \pm 24.14$ & $0.297 \pm 0.026$ & $-1.21 \pm 0.116$ \\
4 & $164.6 \pm 36.61$ & $0.218 \pm 0.007$ & $-1.49 \pm 0.282$ \\
$4 \mathrm{RSV}$ & $225.8 \pm 40.99$ & $0.365 \pm 0.023$ & $-1.41 \pm 0.234$ \\
5 & $172.4 \pm 37.77$ & $0.244 \pm 0.002$ & $-1.84 \pm 0.195$ \\
6 & $236.5 \pm 37.10$ & $0.278 \pm 0.016$ & $-1.65 \pm 0.402$ \\
7 & $380.9 \pm 84.72$ & $0.241 \pm 0.019$ & $-1.56 \pm 0.604$ \\
$7 \mathrm{RSV}$ & $386.82 \pm 26.14$ & $0.386 \pm 0.015$ & $-1.55 \pm 0.786$ \\
9 & $620.8 \pm 330.3$ & $0.472 \pm 0.043$ & $-1.79 \pm 0.144$ \\
10 & $530.0 \pm 295.6$ & $0.469 \pm 0.077$ & $-2.25 \pm 0.712$ \\
11 & $547.5 \pm 432.8$ & $0.542 \pm 0.060$ & $-1.89 \pm 0.740$ \\
12 & $783.1 \pm 213.1$ & $0.597 \pm 0.023$ & $-1.82 \pm 0.497$ \\
\hline
\end{tabular}




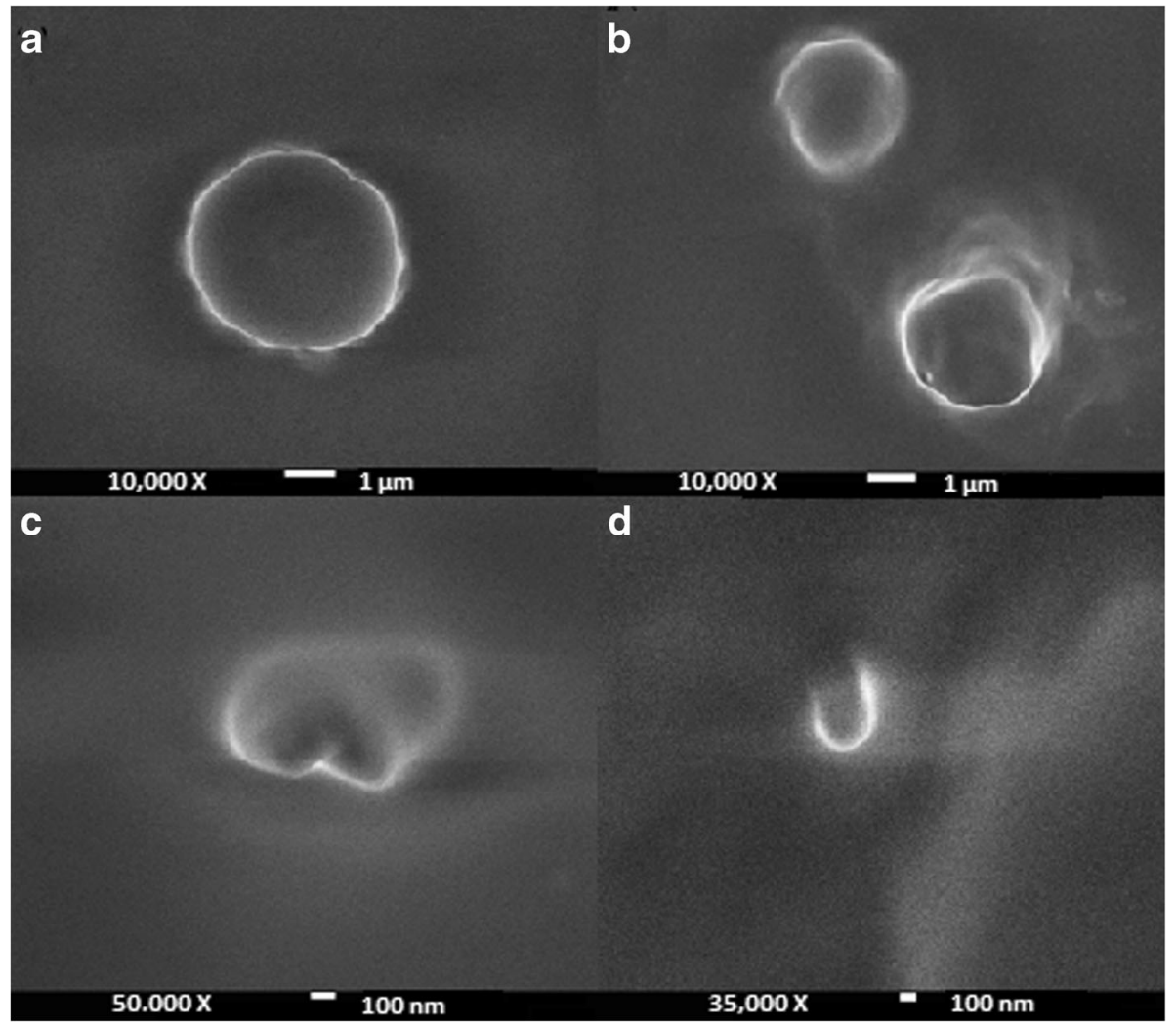

Fig. 1. SEM microphotographs of formulations blank NLC-4 (a), RSV-loaded NLC-4 (b), blank NLC-7 (c), and RSV-loaded NLC-7 (d)

with different materials were reported with similar characteristics [19]. Conversely, formulations prepared with glyceryl behenate presented structures with irregular shape, probably due to its higher instability.

DSC was employed to elucidate details of drug loaded into nanocarriers, if crystalline or amorphous, and the technique also provides structural information of the dispersed particles, given that structural alterations are accompanied by heat exchanges $[33,34]$. Whereas crystalline

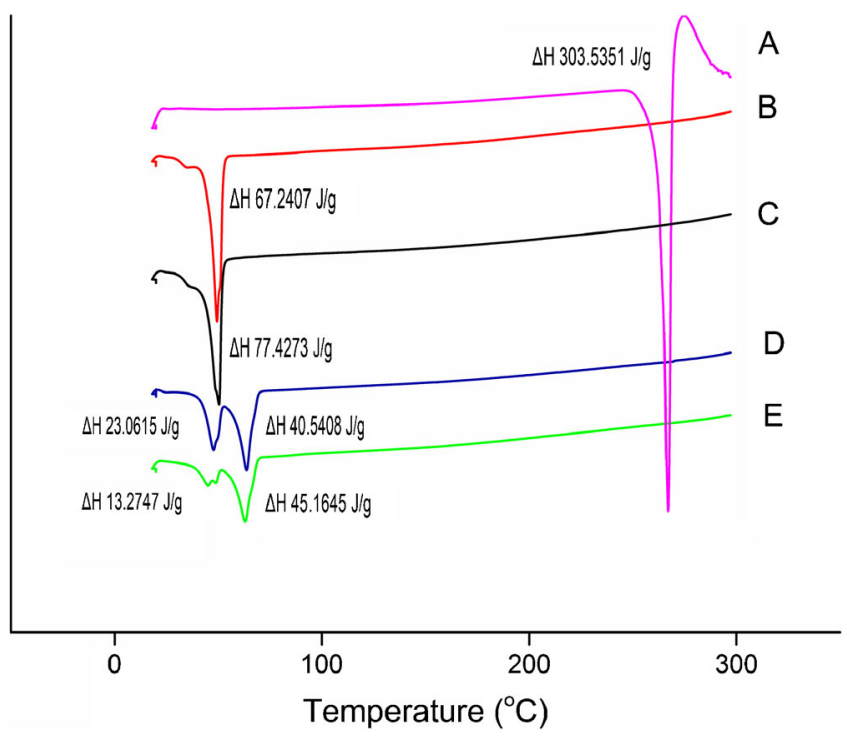

Fig. 2. DSC characterization. A-E RSV, blank NLC-4, RSV-loaded NLC-4, blank NLC-7, and RSV-loaded NLC-7, respectively materials are associated with a well-defined endothermic peak, amorphous materials show a phase transition, equivalent to a more broadened DSC peak [35].

In Fig. 2, DSC curves of RSV, blank NLCs, and RSVloaded NLCs were shown. RSV showed an endothermic peak at $266.47{ }^{\circ} \mathrm{C}(\Delta \mathrm{H} 303.5351 \mathrm{~J} / \mathrm{g})$, in agreement with previous observations reporting its crystalline nature [36]. DSC curves of both blank and RSV-loaded formulations were similar, regarding both their endothermic peaks and the enthalpy values. The fact that RSV endothermic peak was not present could mean that the drug is present in the amorphous state or

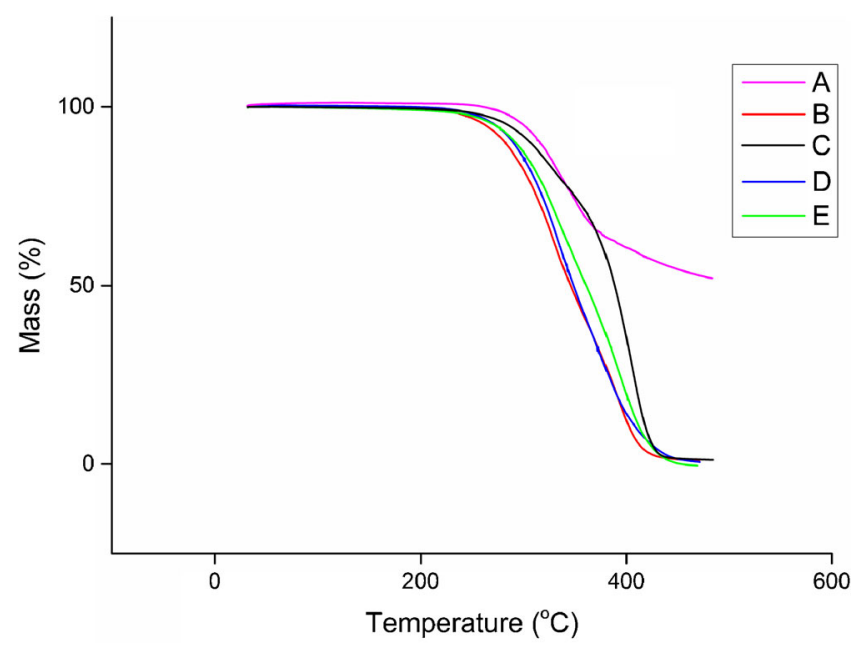

Fig. 3. TGA characterization. A-E RSV, blank NLC-4, RSV-loaded NLC-4, blank NLC-7, and RSV-loaded NLC-7, respectively 


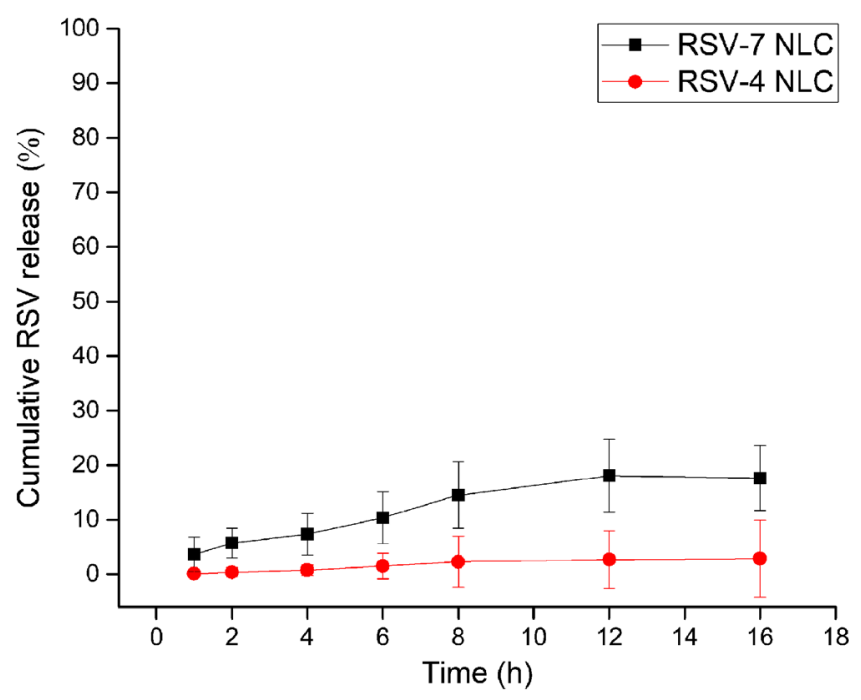

Fig. 4. RSV-loaded NLC 4 (a) and NLC 7 (b) release profile evaluated using modified diffusion cells method until $16 \mathrm{~h}$ of analyses

present as a molecular dispersion. Moreover, it is possible that the surfactants may have dissolved the drug, therefore corroborating the hypothesis of molecular dispersion [37]. In this context, NLCs have been previously prepared with cetyl palmitate exhibited decrease in melting enthalpy suggesting a lower level organization in crystal lattice after RSV incorporation [19].

TGA analysis is used for nanoparticle characterization and indicates degradation behavior of formulations [38]. Herein, we found that RSV has undergone thermal degradation after approximately $250{ }^{\circ} \mathrm{C}$, similarly to previous observations [39]. In a subsequent experiment (data not shown), the drug was heated until $900{ }^{\circ} \mathrm{C}$ and only $10 \%$ of mass remained. But the curve did not finish in a constant mass either. Blank NLC-4 and NLC-7 presented similar behavior, with thermal degradation occurring between approximately 220 and $400{ }^{\circ} \mathrm{C}$, meaning that the blank formulations were thermally more unstable than RSV. In comparison, RSV-loaded NLC-4 formulation had a shift in its TGA curve, which is likely to be correlated with the higher amount of drug loaded (Fig. 3).

In vitro release studies play an important role on the investigation and establishment of product behavior during the various stages of product development. Furthermore, they can reveal information on the dosage form behavior and drug release kinetics. For complex dosage forms, such as nanoparticles, in vitro release assays assume greater significance [40].

Table III. Kinetics Evaluation of RSV Release from NLCs

\begin{tabular}{lcc}
\hline Kinetic modeling & \multicolumn{2}{r}{$r^{2}$ value } \\
\cline { 2 - 3 } & NLC 04 & NLC 07 \\
\hline Zero order & 0.9743 & 0.9726 \\
First order & 0.9131 & 0.9817 \\
Higuchi & 0.6798 & 0.8505 \\
Korsmeyer-Peppas & 0.9374 & 0.9782 \\
Weibull & 0.9861 & 0.9907 \\
\hline
\end{tabular}

Table IV. Halo Inhibition of Formulations in Agar Overlay Diffusion Test

\begin{tabular}{ll}
\hline Formulation & Halo \pm SD $(\mathrm{cm})$ \\
\hline Blank NLC4 & $0.57 \pm 0.058$ \\
RSV-loaded NLC4 & $0.97 \pm 0.058$ \\
Blank NLC7 & $0.73 \pm 0.058$ \\
RSV-loaded NLC7 & $1.07 \pm 0.058$ \\
\hline
\end{tabular}

It is known that drug release from nanoparticles can follow different mechanisms, depending on their composition, including matrix erosion, drug release from nanoparticle surface, and drug diffusion from nanoparticle matrix [41] Hence, solubility, diffusion, and biodegradation of the particle matrix govern the release process. Nanoparticles advantageously allow for drug-sustained release within the target site and the release profile is affected by the incorporation method of drug into the formulation and depends on the type of carriers and the drug physicochemical properties [42].

Both formulations presented very low RSV release, particularly the PEG-40-based nanocarrier, equivalent to $2.67 \%$, compared with $17.67 \%$ obtained with the nanocarrier containing glyceryl behenate (Fig. 4), which occurred due to the crystallinity degree of lipid matrix, in agreement with the DSC data. In a previous paper on RSV-loaded NLCs prepared with different materials, authors suggested that drug release might be dependent on the crystallinity degree of lipid matrix and the presence of the liquid lipid in NLC resulted on less-ordered matrix which better accommodated drug within the nanostructure, preventing its expulsion. On that occasion, NLC released around $8 \%$ of RSV after $24 \mathrm{~h}$, which is comparable with the release profile of NLCs reported in the present paper [19]. Thus, herein, RSV might have stronger affinity with PEG-40 stearate-based NLCs than with formulations prepared with glyceryl behenate, which explains the slower release. Regarding the dissolution profile of the pure drug, recently it was shown that encapsulated RSV had threefold higher dissolution than pure drug [43].

Some models are useful to describe the drug release kinetics from nanoparticles, including the zero order, first order, Higuchi, Korsmeyer-Peppas, and Weibull models. For nondegradable dosage forms that release the same amount of drug over time, zero order is commonly achieved and is desirable, considering the slow release suitable for prolonged action [44]. First-order kinetics is applied for dosage forms which release drug proportionally to the remaining amount. Higuchi model is often employed for lipophilic and hydrophilic drugs that diffuse through non-swellable matrices [45]. Korsmeyer and Peppas kinetics model, however, is based on release combining Fickinian diffusion and non-Fickinian transport [46]. Finally, the Weidbull method is successfully applied for various release profiles and it was the model that best fitted for RSV-loaded NLCs (Table III). This model suggests a complex release mechanism, involving erosion and relaxation of matrix network, which might be due to the presence of Poloxamer 407 which is able to jellify at $37^{\circ} \mathrm{C}$ [47].

Biocompatibility is represented by the ability of a formulation to accomplish its intended function without inducing a negative response in a host organism, in order to 


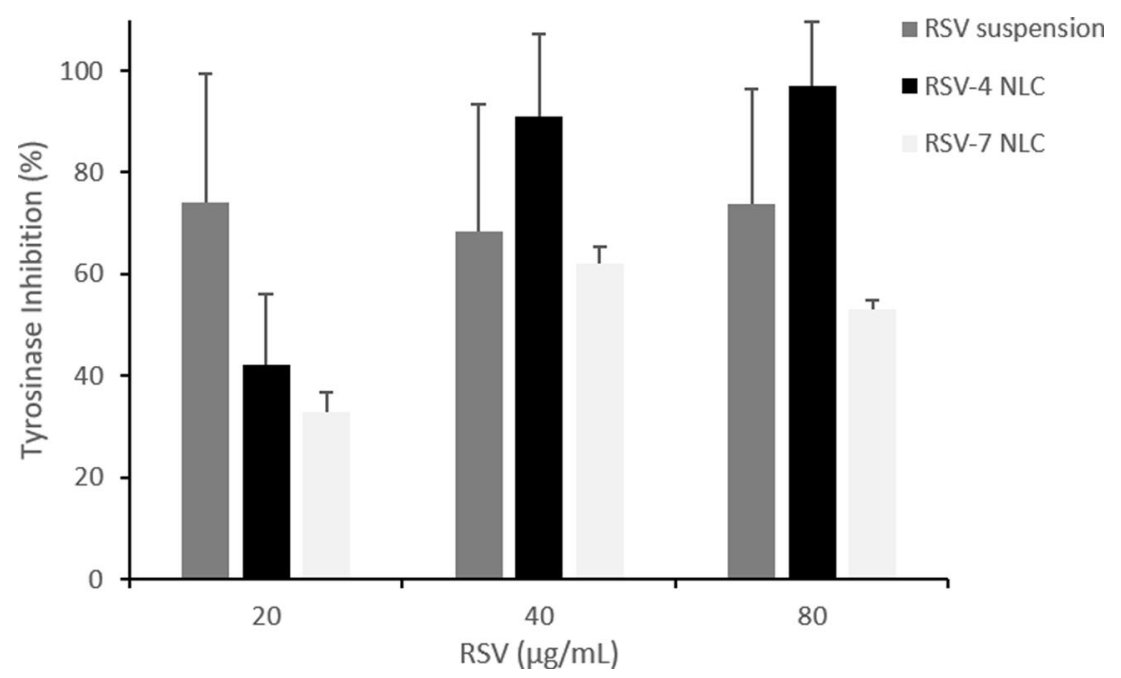

Fig. 5. Tyrosinase inhibition assay of RSV, RSV-4, NLC, and RSV-7, NLC, employing enzyme at $480 \mathrm{IU} / \mathrm{mL} . * p \quad 0.05$

ensure its safety and tolerance to the body. The ISO 10993-5 guideline represents the standard for such in vitro biocompatibility determination. In this context, the agar overlay diffusion test, a qualitative method included in this guideline, allows to measure cytotoxicity by indirect contact. Herein, L929 cell line was employed herein for cytotoxicity evaluation, a very important study of cosmetic formulations [48]. Results showed that all groups tested, blank nanoparticles, and RSVloaded nanoparticles caused moderate cell toxicity in terms of halo inhibition growth (inhibition halo $1 \mathrm{~cm}$ ). Although not causing severe cytotoxicity, it seemed that RSV-loaded formulations presented greater halo inhibition (Table IV). Quantitative evaluation of formulations cytotoxicity could be assessed in future studies; however, the viscosity of NLCs could be a potential influencing factor, creating the need of using high formulation dilutions, which could lead to inaccurate observations. In this context, Rigon et al. [18] evaluated RSV-loaded solid lipid nanoparticles using the quantitative 3(4,5-dimethylthiazol-2-yl)-2,5-diphenyltetrazolium bromide (MTT) method. Although most formulations were regarded as presenting low cytotoxicity, some formulations presented higher cytotoxicity owing to their high viscosity, promoting insufficient oxygen supply and hypoxia. The agar overlay diffusion test, employed herein, could be an option for viscous formulations due to indirect contact between the formulations and the cells. Furthermore, in a previous paper, the agar overlay diffusion test and the MTT assay were used to evaluate the cytotoxicity of $\mathrm{Fe}_{3} \mathrm{O}_{4}$ submicron particles, with comparable results [49]. On the other hand, additional studies, following official guidelines, for example, those of the Food and Drug Administration (FDA), are needed to confirm the safety and biocompatibility of formulations intended for cosmetic use.

Tyrosinase is a well-described target for inhibition to achieve skin whitening not only with cosmetic purpose but also for clinical treatment of pigmentary disorders such as melasma or post-inflammatory hyperpigmentation [50]. Within this context, RSV has been reported as a potent tyrosinase inhibitor, through several mechanisms, including inhibition of the catalytic activity, gene expression, and posttranslational maturation of tyrosinase in melanocytes [50-52].
Herein, we used kojic acid as a positive control to inhibit $480 \mathrm{IU} / \mathrm{mL}$ of tyrosinase, resulting in $50 \%$ of enzyme inhibition for kojic acid concentration of $8.66 \mu \mathrm{g} / \mathrm{mL}$. Thereafter, we evaluated tyrosinase inhibition of RSV and RSV-loaded NLCs. Maximum enzyme inhibition for RSV suspension, PEG-40 stearate containing NLC (RSV-4), and behenate glyceryl containing NLC (RSV-7) corresponded, respectively, to $74.18 \pm 25.10,96.88 \pm 12.72$, and $62.20 \pm 3.19$, in percentage. Although, RSV and formulations were revealed to be less potent than kojic acid (data not shown), the RSV-loaded formulation prepared with PEG-40 stearate (RSV-4) was significantly more effective ( $p$ 0.05) than RSV suspension and behenate glyceryl RSV-7 formulation, resulting on greater tyrosinase inhibition, equivalent to 1.31 and 1.83 times higher, respectively (Fig. 4). It is important to consider that RSV is very lipophilic, with water solubility of $3 \mathrm{mg} / 100 \mathrm{~mL}$, and therefore it was herein employed as an aqueous suspension. Thus, considering the very low aqueous solubility of RSV, the poor enzyme inhibition was expected. Regarding other concentrations of RSV, 20 and $40 \mu \mathrm{g} / \mathrm{mL}$, we observed lower tyrosinase inhibition for the formulation RSV-4 NLC, particularly for the $20 \mu \mathrm{g} / \mathrm{mL}$ concentration, indication dose-dependent response. For comparison, the tyrosinase inhibition of RSV-4 NLC increased form $\sim 40$ to $\sim 90 \%$ at 20 and $40 \mu \mathrm{g} / \mathrm{mL}$, respectively.

Similar results were achieved by Rigon et al. [18], who found that the incorporation of RSV in solid lipid nanoparticles enhanced its tyrosinase inhibitory activity. Protection against degradation and sustained release were factors likely responsible from enhanced enzyme inhibiting ability of RSVloaded nanocarriers. Likewise, resveratrol-enriched rice calli was incorporated into nanoparticles and evaluated regarding its depigmentation following topical administration to ultraviolet B (UVB)-stimulated hyperpigmented guinea pig dorsal skin, resulting in repression of UVB-promoted melanin granules, thereby suppressing hyperpigmentation [53]. Moreover, Ryu and co-workers studied the human skin-whitening effects of a RSV derivative, resveratryl triacetate (RTA). The whitening efficacy of a formulation containing $0.4 \%$ RTA was evaluated in an artificial tanning model in human volunteers, resulting in reduction of hyperpigmented spots [52] (Fig. 5). 
Furthermore, it is known that tyrosinase enzyme inhibition has a role on melanogenesis control, hypothetically applied to reduce melanoma resistance to treatment [54]. For instance, the inhibition of melanogenesis by blocking tyrosinase activity has been demonstrated to sensitize melanoma cells toward cytotoxicity of cyclophosphamide, besides enhancing immunotoxic activity of IL-2 activated lymphocytes [55]. Therefore, RSV-loaded nanoparticles could potentially influence the process of reduction of melanoma resistance to treatment, which needs to be further investigated. For in vivo use, the formulation would need to penetrate into the skin, but not permeate, to reach the melanocytes in the basal membrane. In this context, Sato et al., 2007 investigated the skin penetration of kojic acidloaded emulsion and they observed high skin retention of kojic acid, which is commercially employed as a skin lighting formulation for several applications, including for the treatment of melasma [56].

\section{CONCLUSION}

Taken together, the results presented herein demonstrated the successful encapsulation of trans-resveratrol in nanostructured lipid carriers. Compared with glyceryl behenate, polyoxyethylene 40 stearate was shown to produce nanocarriers with smaller particle size and polydispersity, higher drug encapsulation and more sustained release, due the superior emulsifying ability of polyoxyethylene 40 . Furthermore, trans-resveratrol was likely present as a molecular dispersion in nanocarriers. The cytotoxicity of transresveratrol against a keratinocyte cell line was not enhanced when loaded into nanostructured lipid carriers; however, for evaluation of the safety of formulations, in vitro skin penetration/permeation studies should be conducted. Finally, the better results achieved with nanocarriers prepared with polyoxyethylene 40 resulted in higher tyrosinase inhibition, which could be promising for cosmetic use in skin lightening or for the topical treatment of human cutaneous disorders associated with hyperpigmentation or melanogenesis, which need to be addressed in future studies.

Financial Information The authors would like to thank FAPESP (Fundação de Amparo à Pesquisa do Estado de São Paulo) (grants No. 2013/21500-1, 2012/19568-4, 2011/19018-1, and 2011/16888-5) and PADC (Programa de Apoio ao Desenvolvimento Científico)_FCF-UNESP for financial support.

\section{REFERENCES}

1. Shimada E, Aida K, Sugawara T, Hirata T. Inhibitory effect of topical maize glucosylceramide on skin photoaging in UVAirradiated hairless mice. J Oleo Sci [Internet]. 2011;60:321-5. Available from: http://www.ncbi.nlm.nih.gov/pubmed/21606620

2. Briganti S, Camera E, Picardo M. Chemical and instrumental approaches to treat hyperpigmentation. Pigment Cell Res [Internet]. 2003;16(2):101-10. https://doi.org/10.1034/j.16000749.2003.00029.x.
3. Ndiaye M, Philippe C, Mukhtar H, Ahmad N. The grape antioxidant resveratrol for skin disorders: promise, prospects, and challenges. Arch Biochem Biophys. 2011;508(2):164-70. https://doi.org/10.1016/j.abb.2010.12.030.

4. Williams KA, Kolappswamy K, DeTolla LJ, Vucenik I. Effect of inositol hexaphosphate against UVB damage in HaCaT cells and skin carcinogenesis in SKH1 hairless mice. Comp Med. 2011;61:39-44.

5. Van Nieuwpoort F, Smit NPM, Kolb R, Van Der Meulen H, Koerten H, Pavel S. Tyrosine-induced melanogenesis shows differences in morphologic and melanogenic preferences of melanosomes from light and dark skin types. J Invest Dermatol [Internet]. 2004;122(5):1251-5. https://doi.org/10.1111/j.0022202X.2004.22533.x.

6. Jimbow K, Minamitsuji Y. Topical therapies for melasma and disorders of hyperpigmentation. Dermatol Ther. 2001;14(1):3545. https://doi.org/10.1046/j.1529-8019.2001.014001035.x.

7. Satooka H, Kubo I. Resveratrol as a k cat type inhibitor for tyrosinase: potentiated melanogenesis inhibitor. Bioorganic Med Chem [Internet]. 2012;20(2):1090-9. https://doi.org/ 10.1016/j.bmc.2011.11.030.

8. Cottart $\mathrm{CH}$, Nivet-Antoine V, Laguillier-Morizot C, Beaudeux JL. Resveratrol bioavailability and toxicity in humans. Mol Nutr Food Res. 2010;54(1):7-16. https://doi.org/10.1002/ mnfr.200900437.

9. Hung C-F, Lin Y-K, Huang Z-R, Fang J-Y. Delivery of resveratrol, a red wine polyphenol, from solutions and hydrogels via the skin. Biol Pharm Bull. 2008;31(5):955-62. https://doi.org/10.1248/bpb.31.955.

10. Schaffazick SR, Guterres SS, De Lucca Freitas L, Pohlmann AR. Caracterização e estabilidade físico-química de sistemas poliméricos nanoparticulados para administração de fármacos. Quim Nova. 2003;26(5):726-37. https://doi.org/10.1590/S010040422003000500017.

11. Müller RH, Petersen RD, Hommoss A, Pardeike J. Nanostructured lipid carriers (NLC) in cosmetic dermal products. Adv Drug Deliv Rev. 2007;59(6):522-30. https://doi.org/10.1016/ j.addr.2007.04.012.

12. Gokce EH, Korkmaz E, Dellera E, Sandri G, Cristina Bonferoni M, Ozer O. Resveratrol-loaded solid lipid nanoparticles versus nanostructured lipid carriers: evaluation of antioxidant potential for dermal applications. Int J Nanomedicine. 2012;7:1841-50. https://doi.org/10.2147/IJN.S29710.

13. Jose S, Anju SS, Cinu TA, Aleykutty NA, Thomas S, Souto EB. In vivo pharmacokinetics and biodistribution of resveratrolloaded solid lipid nanoparticles for brain delivery. Int. J. Pharm. 2014;474:6-13. https://doi.org/10.1016/j.ijpharm.2014.08.003.

14. Pandita D, Kumar S, Poonia N, Lather V. Solid lipid nanoparticles enhance oral bioavailability of resveratrol, a natural polyphenol. Food Res Int [Internet]. 2014;62:1165-74. https:// doi.org/10.1016/j.foodres.2014.05.059.

15. Shrotriya SN, Ranpise NS, Vidhate BV. Skin targeting of resveratrol utilizing solid lipid nanoparticle-engrossed gel for chemically induced irritant contact dermatitis. Drug Delivery and Translational Research. 2017;7:37-52.

16. Teskač K, Kristl J. The evidence for solid lipid nanoparticles mediated cell uptake of resveratrol. Int J Pharm. 2010;390(1):61-9. https://doi.org/10.1016/j.ijpharm.2009.10.011.

17. Friedrich RB, Kann B, Coradini K, Offerhaus HL, Beck RCR, Windbergs M. Skin penetration behavior of lipid-core nanocapsules for simultaneous delivery of resveratrol and curcumin. Eur J Pharm Sci [Internet]. 2015;78:204-13. https:// doi.org/10.1016/j.ejps.2015.07.018.

18. Rigon RB, Fachinetti N, Severino P, Santana MHA Skin delivery and in vitro biological evaluation of trans-resveratrolloaded solid lipid. Nanoparticles 2016;1-14.

19. Neves AR, Lúcio M, Martins S, Lima JLC, Reis S. Novel resveratrol nanodelivery systems based on lipid nanoparticles to enhance its oral bioavailability. Int J Nanomedicine. 2013;8:17787. https://doi.org/10.2147/IJN.S37840.

20. Barbosa JP, Neves AR, Silva AM, Barbosa MA, Salette Reis M, Santos SG. Nanostructured lipid carriers loaded with resveratrol modulate human dendritic cells. Int J Nanomedicine. 2016;11:3501-16. https://doi.org/10.2147/IJN.S108694. 
21. Cassano R, Ferrarelli T, Mauro MV, Cavalcanti P, Picci N, Trombino S. Preparation, characterization and in vitro activities evaluation of solid lipid nanoparticles based on PEG-40 stearate for antifungal drugs vaginal delivery. Drug Deliv. 2014;7544:1-10.

22. Negi JS, Chattopadhyay P, Sharma AK, Ram V. Development of solid lipid nanoparticles (SLNs) of lopinavir using hot self nano-emulsification (SNE) technique. Eur J Pharm Sci. 2013;48:231-9. https://doi.org/10.1016/j.ejps.2012.10.022

23. Negi JS, Chattopadhyay P, Sharma AK, Ram V. Development and evaluation of glyceryl behenate based solid lipid nanoparticles (SLNs) using hot self-nanoemulsification (SNE) technique. Arch Pharm Res. 2014;37(3):361-70. https://doi.org/ 10.1007/s12272-013-0154-y.

24. Sato MR, Oshiro Junior JA, Machado RT, de Souza PC, Campos DL, Pavan FR, et al. Nanostructured lipid carriers for incorporation of copper(II) complexes to be used against Mycobacterium tuberculosis. Drug Des Devel Ther [Internet]. 2017;11:909-21. Available from: https://www.dovepress.com/ nanostructured-lipid-carriers-for-incorporation-of-copperii-complexes-peer-reviewed-article-DDDT

25. Eloy JO, Petrilli R, Fernando J, Marcelo H, Antonio R, Palma $\mathrm{J}$, et al. Biointerfaces co-loaded paclitaxel/rapamycin liposomes: development, characterization and in vitro and in vivo evaluation for breast cancer therapy. Colloids and Surfaces B Biointerfaces [Internet]. 2016;141:74-82. https://doi.org/10.1016/ j.colsurfb.2016.01.032.

26. Kobayashi Y, Kayahara H, Tadasa K, Nakamura T, Tanaka H. Synthesis of amino acid derivatives of Kojic acid and their tyrosinase inhibitory activity. Biosci Biotechnol Biochem [Internet]. 1995;59(9):1745-6. https://doi.org/10.1271/bbb.59.1745.

27. Li W, Zhou J, Xu Y. Study of the in vitro cytotoxicity testing of medical devices (review). Biomed Reports 2015;617-20. Doi: https://doi.org/10.3892/br.2015.481

28. Abd-Elbary A, Tadros MI, Alaa-Eldin AA. Sucrose stearateenriched lipid matrix tablets of etodolac: modulation of drug release, diffusional modeling and structure elucidation studies. AAPS PharmSciTech. 2013;14:656-68. https://doi.org/10.1208/ s12249-013-9951-3.

29. Krause B, Mende M, Pötschke P, Petzold G. Dispersability and particle size distribution of CNTs in an aqueous surfactant dispersion as a function of ultrasonic treatment time. Carbon NY. 2010;48:2746-54.

30. Hu FQ, Jiang SP, Du YZ, Yuan H, Ye YQ, Zeng S. Preparation and characterization of stearic acid nanostructured lipid carriers by solvent diffusion method in an aqueous system. Colloids Surf B Biointerfaces. 2005;45(3-4):167-73. https://doi.org/10.1016/ j.colsurfb.2005.08.005.

31. Lourenco C, Teixeira M, Simões S, Gaspar R. Steric stabilization: size and surface properties. Int J Pharm. 1996;138:1-12.

32. Jain D, Athawale R, Bajaj A, Shrikhande S, Goel PN, Gude RP. Biointerfaces studies on stabilization mechanism and stealth effect of poloxamer 188 onto PLGA nanoparticles. Colloids Surf B. 2013;109:59-67.

33. De Mello VA, Ricci-Júnior E. Encapsulation of naproxen in nanostructured system: structural characterization and in vitro release studies. Quim Nova. 2011;34(6):933-9. https://doi.org/ 10.1590/S0100-40422011000600004.

34. Bunjes H, Unruh T. Characterization of lipid nanoparticles by differential scanning calorimetry, X-ray and neutron scattering. Adv Drug Deliv Rev. 2007;59(6):379-402. https://doi.org/ 10.1016/j.addr.2007.04.013.

35. Eloy JO, Marchetti JM. Solid dispersions containing ursolic acid in Poloxamer 407 and PEG 6000: a comparative study of fusion and solvent methods. Powder Technol [Internet]. 2014;253:98106. https://doi.org/10.1016/j.powtec.2013.11.017.

36. Ansari KA, Vavia PR, Trotta F, Cavalli R. Cyclodextrin-based nanosponges for delivery of resveratrol: in vitro characterisation, stability, cytotoxicity and permeation study. AAPS PharmSciTech [Internet]. 2011;12:279-86. https://doi.org/ 10.1208/s12249-011-9584-3.

37. Lira AM, Arajo AAS, Baslio IDJ, Santos BLL, Santana DP, Macedo RO. Compatibility studies of lapachol with pharmaceutical excipients for the development of topical formulations. Thermochim Acta. 2007;457(1-2):1-6. https:// doi.org/10.1016/j.tca.2007.02.017.

38. Omwoyo WN, Ogutu B, Oloo F, Swai H, Kalombo L, Melariri $\mathrm{P}$, et al. Preparation, characterization, and optimization of primaquine-loaded solid lipid nanoparticles. Int J Nanomedicine. 2014;9:3865-74. https://doi.org/10.2147/ IJN.S62630.

39. Sanna V, Siddiqui IA, Sechi M, Mukhtar H. Resveratrol-loaded nanoparticles based on poly(epsilon-caprolactone) and poly(d,1lactic-co-glycolic acid)-poly(ethylene glycol) blend for prostate cancer treatment. Mol Pharm. 2013;10:3871-81. Available from: http://www.ncbi.nlm.nih.gov/pubmed/23968375

40. Souza SD. A review of in vitro drug release test methods for nano-sized dosage forms. Adv Pharmaceutics. 2014.

41. Agnihotri SA, Mallikarjuna NN, Aminabhavi TM. Recent advances on chitosan-based micro- and nanoparticles in drug delivery. J Control Release. 2004;100(1):5-28. https://doi.org/ 10.1016/j.jconrel.2004.08.010.

42. Singh R, Lillard W. Nanoparticle-based targeted drug delivery. Exp Mol Pathol. 2009;86:215-23.

43. Juère E, Florek J, Bouchoucha M, Jambhrunkar S, Wong KY, Popat A, et al. In vitro dissolution, cellular membrane permeability and anti-inflammatory response of resveratrolencapsulated mesoporous silica nanoparticles. Mol Pharm [Internet]. 2017; acs.molpharmaceut.7b00529; https://doi.org/ 10.1021/acs.molpharmaceut.7b00529.

44. Costa P, Lobo JMS. Modelling and comparison of dissolution profile. Eur J Pharm Sci. 2001;13:123-33.

45. Higuchi T. Mechanism of sustained-action medication. J Pharm Sci. 1963;52:1145-9.

46. Korsmeyer RW, Gurny R, Doelker E, Buri P, Peppas NA. Mechanisms of solute release from porous hydrophilic polymers. Int J Pharm. 1983;15(1):25-35. https://doi.org/10.1016/ 0378-5173(83)90064-9.

47. Schmolka IR. Artificial skin. I. Preparation and properties treatment of burns. J Biomed Mater Res. 1972;6:571-82.

48. Rodrigues F, Gaspar C, Palmeira-de-Oliveira A, Sarmento B, Helena Amaral M, Oliveira MB. Application of coffee silverskin in cosmetic formulations: physical/antioxidant stability studies and cytotoxicity effects. Drug Dev Ind Pharm [Internet]. 2015;0:1-8. https://doi.org/10.3109/03639045.2015.1035279.

49. Kim DH, Lee SH, Kim KN, Kim KM, Shim IB, Lee YK. Cytotoxicity of ferrite particles by MTT and agar diffusion methods for hyperthermic application. J Magn Magn Mater. 2005;293(1):287-92. https://doi.org/10.1016/j.jmmm.2005.02.078.

50. Smit N, Vicanova J, Pavel S. The hunt for natural skin whitening agents. Int J Mol Sci. 2009;10:5326-49.

51. Bernard P, Berthon JY. Resveratrol: an original mechanism on tyrosinase inhibition. Int J Cosmet Sci. 2000;22(3):219-26. https://doi.org/10.1046/j.1467-2494.2000.00019.x.

52. Ryu JH, Seok JK, An SM, Baek JH, Koh JS, Boo YC. A study of the human skin-whitening effects of resveratryl triacetate. Arch Dermatol Res. 2015;307(3):239-47. https://doi.org/10.1007/ s00403-015-1556-0.

53. Lee TH, Kang JH, Seo JO, Baek SH, Moh SH, Chae JK, et al. Anti-melanogenic potentials of nanoparticles from calli of resveratrol-enriched rice against UVB-induced hyperpigmentation in guinea pig skin. Biomol Ther. 2016;24(1):85-93. https:// doi.org/10.4062/biomolther.2015.165.

54. Huang Y, Vecchio D, Avci P, Yin R, Garcia-diaz M, Hamblin MR. Melanoma resistance to photodynamic therapy: new insights. J Biol Chem. 2013;394:239-50.

55. Slominski A, Zbytek B, Slominski R. Inhibitors of melanogenesis increase toxicity of cyclophosphamide and lymphocytes against melanoma cells. Int J Cancer [Internet]. 2009;124:14707. Available from: http://www.ncbi.nlm.nih.gov/pubmed/ 19085934

56. Sato MEO, Gomara F, Pontarolo R, Andreazza IF, Zaroni M. Permeação cutânea in vitro do ácido kójico. Rev Bras Ciências Farm. 2007;43(2):195-203. https://doi.org/10.1590/S151693322007000200005 . 